\title{
Effects of lowly ionized ions on silicon K-shell absorption spectra (Research Note)
}

\author{
H. G. Wei ${ }^{1}$, J. R. Shi ${ }^{1}$, G. Y. Liang ${ }^{1}$, F. L. Wang ${ }^{1}$, J. Y. Zhong ${ }^{1,2}$, and G. Zhao ${ }^{1}$ \\ 1 Key Laboratory of Optical Astronomy, National Astronomical Observatories, Chinese Academy of Sciences, 100012 Beijing, \\ PR China \\ e-mail: [whg;gzhao]@nao.cas.cn \\ 2 Department of Astronomy, Beijing Normal University, 100875 Beijing, PR China
}

Received 27 November 2015 / Accepted 23 March 2016

\begin{abstract}
Context. In both astrophysical and laboratory plasmas, K-shell absorption spectra have become powerful diagnostic tools to investigate electron density and temperature. These spectra are also widely used to verify the opacity codes in laboratory settings.

Aims. We report the effects of the low ionization silicon ions, namely from $\mathrm{Si}$ I to Si V, which have rarely been considered in previous models, on the K-shell silicon absorption spectra.

Methods. The Si K-shell atomic data were calculated with the flexible atomic code, which is a fully relativistic atomic program with configuration interaction taken into consideration. Detailed level accounting models were employed to calculate the absorption spectra.

Results. We calculate the Si absorption spectra in local thermodynamic equilibrium conditions with temperature and density ranges of $20-70 \mathrm{eV}$ and $\sim 10^{20} \mathrm{~cm}^{-3}$ to $\sim 10^{22} \mathrm{~cm}^{-3}$, respectively, and show the contributions of the lowly ionized ions to the K-shell absorption spectra of silicon. We also investigate the effects of the different atomic data on the absorption spectra. We find good agreement between our results and these from OPLIB.

Conclusions. We find that the contributions from these lowly ionized ions cannot be neglected at relative low temperatures. Accurate experimental measurements are needed to benchmark the theoretical calculations.
\end{abstract}

Key words. atomic data - opacity - plasmas

\section{Introduction}

K-shell spectra, especially those from helium-like ions, are observed in astrophysical objects (Kaspi et al. 2000; Sako et al. 2001; Krongold et al. 2003; Holczer et al. 2007). Gabriel \& Jordan (1969) firstly pointed out that the K-shell lines, namely the triplet lines, of He-like ions could be used as a diagnostic tool on solar coronal plasmas because these line ratios are sensitive to electron density and temperatures over a wide range (Kallman et al. 2014). Since then, several improvements have been made to this method using more accurate atomic data and taking more detailed atomic process into consideration (Porquet et al. 2001). Not limited to coronal plasmas, which is dominated by electron excitation and ionization, this method can also be applied to photo-ionized plasmas or nonequilibrium plasmas (Porquet et al. 2000, 2010). Numerous high resolution spectra obtained by modern X-ray satellites, such as Chandra, XMM, and Suzaku, have also revealed detailed K-shell features from lowly ionized ions besides He-like ions (Turner \& Miller 2009; Kallman et al. 2014). These lines could provide valuable information regarding these astrophysical objects, such as outflow velocity, temperature, and chemical abundances; they can also be used as diagnostic tools in laboratory plasmas, such as laser plasmas (Bailey et al. 2009), tokamak plasmas (Beiersdorfer et al. 2003), or electron beam ion trap (EBIT) plasmas (Beiersdorfer 2009). Particularly in the experiments of opacity measurements, K-shell spectra are widely used to determine the conditions of the plasma (Bailey et al. 2009; Nagayama et al. 2014). The density is inferred from line Stark broadening, while the electron temperature can be deduced by the K-shell absorption spectra because the K-shell absorption features have a strong dependence on this temperature.

Comprehensive and accurate atomic data are essential to analyze the K-shell spectra, however, K-shell atomic data from low ionization ions with an occupied M-shell are rarely considered in previous work. For example, Sim et al. (2010) included the $\mathrm{M}$-shell ions for $\mathrm{Fe}$ and $\mathrm{Ni}$, while only including L-shell ions for $\mathrm{Mg}, \mathrm{Si}, \mathrm{S}, \mathrm{Ar}$, and $\mathrm{Ca}$ in modeling the X-ray spectra for active galactic nuclei (AGN) outflows. And in laboratory plasmas, usually only K-shell and L-shell ions are considered when modeling the K-shell spectra from opacity measurements (Wei et al. 2008; Bailey et al. 2009). By including the inner shell atomic data in the opacity calculations, Badnell et al. $(2004,2005)$ reported that the Rosseland-mean opacities from OP are in good agreement with those from OPAL (Rogers \& Iglesias 1992). Nevertheless, the K-shell atomic data for M-shell ions are not included in their updated work. Recently, experimental results from Bailey et al. (2015) revealed that some transitions are omitted in OP. This omission may account for the discrepancies between the measured opacities and those from theoretical models. Lack of $\mathrm{K}$-shell atomic data is the main reason for the current ignorance concerning M-shell ions.

Fortunately, since the launch of high resolution X-ray satellites (Chandra and XMM), K-shell atomic data have been extensively calculated (Palmeri et al. 2002, 2003, 2008, 2011, 2012; Behar \& Netzer 2002; Bautista et al. 2003, 2004; Mendoza et al. 2004; Witthoeft et al. 2009; Wei et al. 2010, 2014). Among these 
works, Palmeri et al. (2008) reported firstly the K-shell data for M-shell ions of $\mathrm{Ne}, \mathrm{Mg}, \mathrm{Si}, \mathrm{S}, \mathrm{Ar}$, and $\mathrm{Ca}$ with the codes HFR (Cowan 1981) and AUTOSTRUCTURE (Badnell 1986, 1997). In this note, we use the example of silicon to show the effects of the low ionized silicon ions, namely silicon M-shell ions, on the silicon absorption spectra. For simplicity, we calculated the spectra under local thermodynamic equilibrium (LTE) conditions, which are widely achieved in stellar opacity calculations and opacity experimental measurements.

\section{Model}

We use the detailed-level-accounting (hereafter, DLA) model to calculate the absorption spectra for silicon plasmas in LTE conditions (Zeng et al. 2004; Jin et al. 2009). Generally, in opacity measurement experiments (Bailey et al. 2009), the plasmas are assumed to be uniform, thus, the spectra after transmission through the plasmas can be written as

$T(v)=e^{-\kappa_{v} L}$,

where $\kappa_{v}$ is the absorption coefficient, while $L$ is the thickness of the plasma. The parameter $\kappa_{v}$ can be written as

$\kappa_{v}=\sum N_{i l} \frac{\pi e^{2} f_{i l u}}{m_{e} c} \phi(v)\left(1-e^{-h v / k T}\right)$,

where $N_{i l}$ is the population density for level $l$ of the ionization state $i, f_{i l u}$ is the oscillator strength from level $l$ to $u, e$ and $m_{e}$ are the charge and mass of the electron, $c$ is the speed of light in a vacuum, and $\phi(v)$ is the line profile function. In our model, the line profile is described by a Voigt profile, which is the convolution of a Gaussian and a Lorentzian profile. The Gaussian profile is composed of the Doppler broadening and instrumental broadening, while the Lorentz profile consists of the radiative and autoionization resonances broadening. The radiative and autoionization widths are adopted from the calculations of Palmeri et al. (2008). We use the same value of the line profile $\phi(v)$ in our models.

In fact, K-shell spectra are used as diagnostic tools mainly because of the presence of $\mathrm{K}$-shell line features, which originate from the bound-bound line transitions (Bailey et al. 2009). The bound-free and free-free absorptions are usually removed from the absorption spectra in laboratory plasmas (Nagayama et al. 2014; Rochau et al. 2005). Identifications of the key line features are also the most important aspects when modeling X-ray spectra from AGN (Turner \& Miller 2009). Therefore, for simplicity, in the present case we concentrate on the contributions of the bound-bound line transitions from the M-shell ions of silicon to the absorption spectra.

In our model, the silicon K-shell atomic data are calculated with the flexible atomic code (FAC), which is developed by $\mathrm{Gu}$ (2003). The FAC is a fully relativistic atomic program that takes configuration interaction into consideration; it is widely used in the calculations of the atomic data needed to analyze X-ray spectra (Beiersdorfer 2009). The accuracy of these calculations were assessed by many recent works (Massacrier \& Artru 2012; Wei et al. 2010). In order to investigate the effects of different atomic data on the calculated absorption spectra, we adopted the same configurations as those in the work of Palmeri et al. (2008), who calculated the K-shell atomic data. For Be-like and C-like ions, the accuracy of the K-shell data was described in our previous work (Wei et al. 2010, 2014). The energy levels are generally accurate to a few eV (less than $4 \mathrm{eV}$ ), the wavelengths to within $15 \mathrm{~m} \AA$, and the radiation to around $20 \%$ for most transitions.

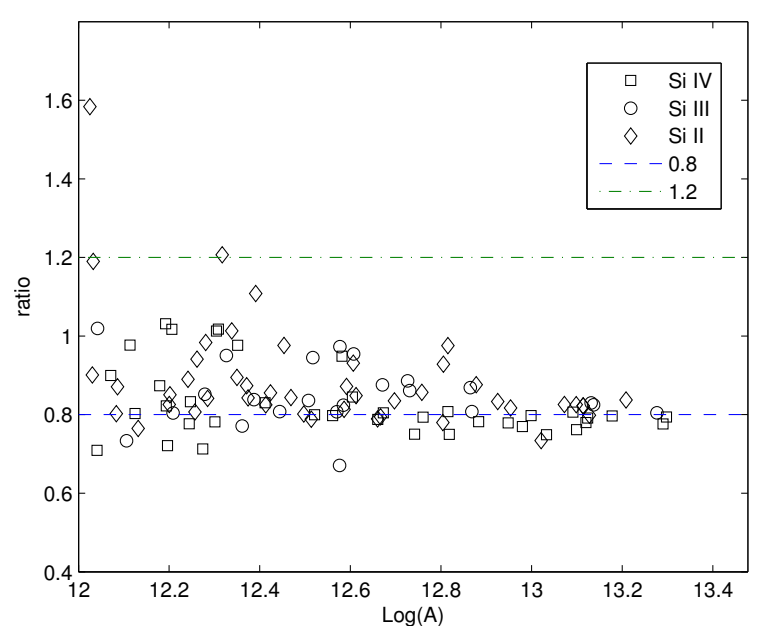

Fig. 1. Comparison of the present FAC radiative rates with the HFR calculations for Si II, Si III, and Si IV.

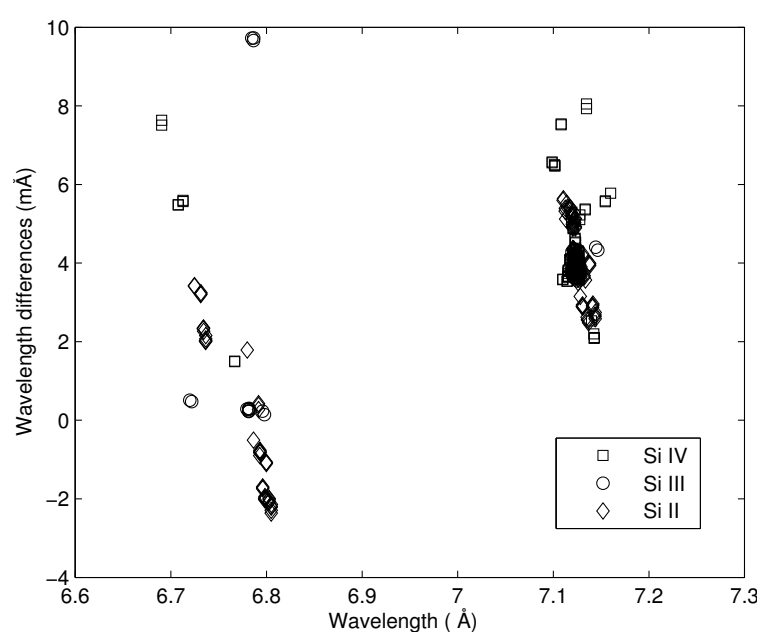

Fig. 2. Comparison of the present FAC wavelengths with the HFR calculations for Si II, Si III, and Si IV.

We provide a detailed comparison between our FAC results and those from Palmeri et al. (2008) of the atomic data for Si V and Si VI in Table 1. The wavelength differences are also less than $15 \mathrm{~mA}$. For $A$ values, the present FAC results are also in agreement with those from HFR by about $20 \%$. Figure 1 shows the comparison of radiative rates $\left(>1 E+12 s^{-1}\right)$ between our present work and those from HFR (Palmeri et al. 2008) for Si II, Si III, and Si IV, and the agreement is also within 20\%. In Fig. 2 we show the wavelength differences between our FAC data and those from HFR for Si II, Si III, and Si IV; they are less than $10 \mathrm{~m} \AA$. In general, these comparisons indicated the present accuracy of our atomic data.

\section{Results and discussions}

In laboratory, opacity can be measured with the plasma temperature range from a few eV to several hundred $\mathrm{eV}$ (Bailey et al. 2009). For iron, the plasma temperatures are around $20 \mathrm{eV}$ in early opacity experiments using large laser facilities (Da silva et al. 1992; Winhart et al. 1995, 1996; ChenaisPopovics et al. 2000). In experiments by Springer et al. (1992) and Zhang et al. (2012), they measured temperatures up to 50 to $76 \mathrm{eV}$. Very recently, Bailey et al. $(2007,2015)$ made a break through in the temperature (150 to $210 \mathrm{eV}$ ) and density (up to 
Table 1. Comparison of the atomic data for Si V and Si VI.

\begin{tabular}{|c|c|c|c|c|c|c|}
\hline Ion & Upper level & Lower level & $\begin{array}{c}\lambda(F A C) \\
\AA\end{array}$ & $\begin{array}{c}\lambda(H F R) \\
\AA\end{array}$ & $\begin{array}{c}A_{u l}(F A C) \\
\left(\mathrm{s}^{-1}\right)\end{array}$ & $\begin{array}{c}A_{u l}(H F R) \\
\left(\mathrm{s}^{-1}\right)\end{array}$ \\
\hline \multirow[t]{2}{*}{ Si VI } & $1 s 2 s^{2} 2 p^{6}{ }^{2} S_{1 / 2}$ & $1 s^{2} 2 s^{2} 2 p^{5}{ }^{3} \mathrm{P}_{3 / 2}^{o}$ & 7.110 & 7.122 & $2.32 \mathrm{E}+13$ & $2.44 \mathrm{E}+13$ \\
\hline & $1 s 2 s^{2} 2 p^{6}{ }^{2} S_{1 / 2}$ & $1 \mathrm{~s}^{2} 2 \mathrm{~s}^{2} 2 \mathrm{p}^{5}{ }^{3} \mathrm{P}_{1 / 2}^{\mathrm{o}}$ & 7.113 & 7.125 & $1.17 \mathrm{E}+13$ & $1.22 \mathrm{E}+13$ \\
\hline \multirow[t]{37}{*}{ Si V } & $1 s 2 s^{2} 2 p^{6} 3 s^{3} S_{1}$ & $1 s^{2} 2 s^{2} 2 p^{5} 3 s^{3} P_{2}^{o}$ & 7.115 & 7.112 & $1.66 \mathrm{E}+13$ & $2.10 \mathrm{E}+13$ \\
\hline & $1 s 2 s^{2} 2 p^{6} 3 s^{3} S_{1}$ & $1 s^{2} 2 s^{2} 2 p^{5} 3 s^{3} P_{1}^{o}$ & 7.117 & 7.113 & $9.25 \mathrm{E}+12$ & $1.15 E+13$ \\
\hline & $1 s 2 s^{2} 2 p^{6} 3 s^{3} S_{1}$ & $1 s^{2} 2 s^{2} 2 p^{5} 3 s^{3} P_{0}^{o}$ & 7.118 & 7.114 & $3.33 \mathrm{E}+12$ & $4.19 \mathrm{E}+12$ \\
\hline & $1 s 2 s^{2} 2 p^{6} 3 s^{3} S_{1}$ & $1 s^{2} 2 s^{2} 2 p^{5} 3 s^{1} P_{1}^{o}$ & 7.122 & 7.117 & $7.22 \mathrm{E}+11$ & $1.10 \mathrm{E}+12$ \\
\hline & $1 \mathrm{~s} 2 \mathrm{~s}^{2} 2 \mathrm{p}^{6} 3 \mathrm{~s}^{1} \mathrm{~S}_{0}$ & $1 s^{2} 2 s^{2} 2 p^{5} 3 s^{3} P_{1}^{o}$ & 7.111 & 7.108 & $2.15 \mathrm{E}+12$ & $3.32 \mathrm{E}+12$ \\
\hline & $1 \mathrm{~s} 2 \mathrm{~s}^{2} 2 \mathrm{p}^{6} 3 \mathrm{~s}^{1} \mathrm{~S}_{0}$ & $1 s^{2} 2 s^{2} 2 p^{5} 3 s^{1} P_{1}^{o}$ & 7.116 & 7.112 & $2.80 \mathrm{E}+13$ & $3.45 E+13$ \\
\hline & $1 s 2 s^{2} 2 p^{6} 3 p^{3} P_{0}^{o}$ & $1 s^{2} 2 s^{2} 2 p^{5} 3 p^{3} S_{1}$ & 7.111 & 7.106 & $4.62 \mathrm{E}+12$ & $5.61 \mathrm{E}+12$ \\
\hline & $1 s 2 s^{2} 2 p^{6} 3 p^{3} P_{0}^{o}$ & $1 s^{2} 2 s^{2} 2 p^{5} 3 p^{3} D_{1}$ & 7.117 & 7.113 & $2.04 \mathrm{E}+13$ & $2.59 \mathrm{E}+13$ \\
\hline & $1 s 2 s^{2} 2 p^{6} 3 p^{3} P_{0}^{o}$ & $1 s^{2} 2 s^{2} 2 p^{5} 3 p^{1} P_{1}$ & 7.120 & 7.116 & $3.06 \mathrm{E}+10$ & $2.40 \mathrm{E}+09$ \\
\hline & $1 s 2 s^{2} 2 p^{6} 3 p^{3} P_{0}^{o}$ & $1 s^{2} 2 s^{2} 2 p^{5} 3 p^{3} P_{1}$ & 7.121 & 7.117 & $4.82 \mathrm{E}+12$ & $6.18 \mathrm{E}+12$ \\
\hline & $1 s 2 s^{2} 2 p^{6} 3 p^{3} P_{1}^{o}$ & $1 s^{2} 2 s^{2} 2 p^{6}{ }^{1} S_{0}$ & 6.684 & 6.677 & $1.02 \mathrm{E}+10$ & $8.11 \mathrm{E}+09$ \\
\hline & $1 s 2 s^{2} 2 p^{6} 3 p^{3} P_{1}^{o}$ & $1 s^{2} 2 s^{2} 2 p^{5} 3 p^{3} s_{1}$ & 7.111 & 7.106 & $3.73 \mathrm{E}+12$ & $4.66 \mathrm{E}+12$ \\
\hline & $1 s 2 s^{2} 2 p^{6} 3 p^{3} P_{1}^{o}$ & $1 s^{2} 2 s^{2} 2 p^{5} 3 p^{3} D_{2}$ & 7.116 & 7.112 & $1.42 \mathrm{E}+13$ & $1.75 E+13$ \\
\hline & $1 \mathrm{~s} 2 \mathrm{~s}^{2} 2 \mathrm{p}^{6} 3 \mathrm{p}^{3} \mathrm{P}_{1}^{\mathrm{o}}$ & $1 s^{2} 2 s^{2} 2 p^{5} 3 p^{3} D_{1}$ & 7.117 & 7.113 & $2.35 \mathrm{E}+12$ & $2.74 \mathrm{E}+12$ \\
\hline & $1 s 2 s^{2} 2 p^{6} 3 p^{3} P_{1}^{o}$ & $1 s^{2} 2 s^{2} 2 p^{5} 3 p^{3} P_{2}$ & 7.119 & 7.115 & $2.48 \mathrm{E}+12$ & $3.50 \mathrm{E}+12$ \\
\hline & $1 \mathrm{~s} 2 \mathrm{~s}^{2} 2 \mathrm{p}^{6} 3 \mathrm{p}^{3} \mathrm{P}_{1}^{\mathrm{o}}$ & $1 s^{2} 2 s^{2} 2 p^{5} 3 p^{1} P_{1}$ & 7.120 & 7.116 & $2.83 \mathrm{E}+12$ & $3.72 \mathrm{E}+12$ \\
\hline & $1 s 2 s^{2} 2 p^{6} 3 p^{3} P_{1}^{o}$ & $1 s^{2} 2 s^{2} 2 p^{5} 3 p^{1} D_{2}$ & 7.120 & 7.116 & $5.13 \mathrm{E}+10$ & $6.65 E+10$ \\
\hline & $1 s 2 s^{2} 2 p^{6} 3 p^{3} P_{1}^{o}$ & $1 s^{2} 2 s^{2} 2 p^{5} 3 p^{3} P_{0}$ & 7.121 & 7.117 & $3.25 \mathrm{E}+12$ & $4.10 \mathrm{E}+12$ \\
\hline & $1 s 2 s^{2} 2 p^{6} 3 p^{3} P_{1}^{o}$ & $1 s^{2} 2 s^{2} 2 p^{5} 3 p^{3} P_{1}$ & 7.121 & 7.117 & $9.94 \mathrm{E}+11$ & $1.35 \mathrm{E}+12$ \\
\hline & $1 s 2 s^{2} 2 p^{6} 3 p^{3} P_{1}^{o}$ & $1 s^{2} 2 s^{2} 2 p^{5} 3 p^{1} S_{0}$ & 7.153 & 7.143 & $2.67 \mathrm{E}+10$ & $3.32 \mathrm{E}+10$ \\
\hline & $1 s 2 s^{2} 2 p^{6} 3 p^{3} P_{2}^{o}$ & $1 s^{2} 2 s^{2} 2 p^{5} 3 p^{3} S_{1}$ & 7.111 & 7.105 & $2.62 \mathrm{E}+12$ & $3.35 \mathrm{E}+12$ \\
\hline & $1 s 2 s^{2} 2 p^{6} 3 p^{3} P_{2}^{o}$ & $1 s^{2} 2 s^{2} 2 p^{5} 3 p^{3} D_{3}$ & 7.115 & 7.111 & $1.41 \mathrm{E}+13$ & $1.78 \mathrm{E}+13$ \\
\hline & $1 s 2 s^{2} 2 p^{6} 3 p^{3} P_{2}^{o}$ & $1 s^{2} 2 s^{2} 2 p^{5} 3 p^{3} D_{2}$ & 7.116 & 7.112 & $1.01 \mathrm{E}+12$ & $1.22 \mathrm{E}+12$ \\
\hline & $1 s 2 s^{2} 2 p^{6} 3 p^{3} P_{2}^{o}$ & $1 s^{2} 2 s^{2} 2 p^{5} 3 p^{3} P_{2}$ & 7.119 & 7.115 & $3.88 \mathrm{E}+12$ & $3.99 \mathrm{E}+12$ \\
\hline & $1 s 2 s^{2} 2 p^{6} 3 p^{3} P_{2}^{o}$ & $1 s^{2} 2 s^{2} 2 p^{5} 3 p^{1} P_{1}$ & 7.120 & 7.116 & $1.01 \mathrm{E}+12$ & $1.26 \mathrm{E}+12$ \\
\hline & $1 s 2 s^{2} 2 p^{6} 3 p^{3} P_{2}^{o}$ & $1 s^{2} 2 s^{2} 2 p^{5} 3 p^{1} D_{2}$ & 7.120 & 7.116 & $4.99 \mathrm{E}+12$ & $7.27 \mathrm{E}+12$ \\
\hline & $1 s 2 s^{2} 2 p^{6} 3 p^{3} P_{2}^{o}$ & $1 s^{2} 2 s^{2} 2 p^{5} 3 p^{3} P_{1}$ & 7.121 & 7.117 & $2.24 \mathrm{E}+12$ & $2.77 \mathrm{E}+12$ \\
\hline & $1 s 2 s^{2} 2 p^{6} 3 p^{1} P_{1}^{o}$ & $1 s^{2} 2 s^{2} 2 p^{6}{ }^{1} S_{0}$ & 6.681 & 6.677 & $3.08 \mathrm{E}+12$ & $2.87 \mathrm{E}+12$ \\
\hline & $1 s 2 s^{2} 2 p^{6} 3 p^{1} P_{1}^{o}$ & $1 s^{2} 2 s^{2} 2 p^{5} 3 p^{3} s_{1}$ & 7.107 & 7.106 & $8.19 \mathrm{E}+10$ & $9.20 \mathrm{E}+10$ \\
\hline & $1 s 2 s^{2} 2 p^{6} 3 p^{1} P_{1}^{o}$ & $1 s^{2} 2 s^{2} 2 p^{5} 3 p^{3} D_{2}$ & 7.113 & 7.112 & $9.32 \mathrm{E}+11$ & $1.64 \mathrm{E}+12$ \\
\hline & $1 s 2 s^{2} 2 p^{6} 3 p^{1} P_{1}^{o}$ & $1 s^{2} 2 s^{2} 2 p^{5} 3 p^{3} D_{1}$ & 7.114 & 7.113 & $9.20 \mathrm{E}+11$ & $1.32 \mathrm{E}+12$ \\
\hline & $1 s 2 s^{2} 2 p^{6} 3 p^{1} P_{1}^{o}$ & $1 s^{2} 2 s^{2} 2 p^{5} 3 p^{3} P_{2}$ & 7.116 & 7.115 & $7.48 \mathrm{E}+12$ & $1.07 \mathrm{E}+13$ \\
\hline & $1 s 2 s^{2} 2 p^{6} 3 p^{1} P_{1}^{o}$ & $1 s^{2} 2 s^{2} 2 p^{5} 3 p^{1} P_{1}$ & 7.116 & 7.116 & $5.41 \mathrm{E}+12$ & $6.74 \mathrm{E}+12$ \\
\hline & $1 s 2 s^{2} 2 p^{6} 3 p^{1} P_{1}^{o}$ & $1 s^{2} 2 s^{2} 2 p^{5} 3 p^{1} D_{2}$ & 7.117 & 7.116 & $8.18 \mathrm{E}+12$ & $8.70 \mathrm{E}+12$ \\
\hline & $1 s 2 s^{2} 2 p^{6} 3 p^{1} P_{1}^{o}$ & $1 s^{2} 2 s^{2} 2 p^{5} 3 p^{3} P_{0}$ & 7.117 & 7.117 & $2.64 \mathrm{E}+10$ & $3.41 \mathrm{E}+10$ \\
\hline & $1 s 2 s^{2} 2 p^{6} 3 p^{1} P_{1}^{o}$ & $1 s^{2} 2 s^{2} 2 p^{5} 3 p^{3} P_{1}$ & 7.117 & 7.117 & $3.54 \mathrm{E}+12$ & $4.44 \mathrm{E}+12$ \\
\hline & $1 s 2 s^{2} 2 p^{6} 3 p^{1} P_{1}^{o}$ & $1 s^{2} 2 s^{2} 2 p^{5} 3 p^{1} S_{0}$ & 7.150 & 7.143 & $3.24 \mathrm{E}+12$ & $3.62 \mathrm{E}+12$ \\
\hline
\end{tabular}

Notes. FAC: present FAC calculations. HFR: calculations with HFR (Palmeri et al. 2008).

$\sim 10^{22} \mathrm{~cm}^{-3}$ ) in iron opacity experiments at the Sandia National Laboratory Z-pinch facility, which almost reproduce the conditions of the solar radiation convection boundary. For other light elements, extensive experiments were also conducted in laboratories (Davidson et al. 1988; Perry et al. 1991; Xiong et al. 2016). Most of these above experiments measured the opacity at an order of 0.001 to $0.1 \mathrm{~g} / \mathrm{cm}^{-3}$, which is corresponding to an electron density of order from $\sim 10^{20} \mathrm{~cm}^{-3}$ to $\sim 10^{22} \mathrm{~cm}^{-3}$. Since the lowly ionized silicon ions do not exist at high temperatures, here we only consider temperatures up to several tens eV. In fact, as a result of the time evolution or gradients in density and temperature, plasmas with low temperatures can exist even in high temperatures (Chenais-Popovics et al. 2000). In particular,
Badnell et al. (2005) found that the differences in Rosselandmean opacity between OP and OPAL get larger at low temperatures. It is interesting to test the contributions from the lowly ionized silicon ions to the K-shell spectra and, thus, we limited the temperatures to the range 20 to $70 \mathrm{eV}$, while the densities range between $\sim 10^{20} \mathrm{~cm}^{-3}$ to $\sim 10^{22} \mathrm{~cm}^{-3}$. These two parameters are chosen to cover most of the plasma conditions, namely from high to low temperatures and from high to low densities.

\subsection{Effects of lowly ionized silicon ions}

The K-shell spectra of silicon in LTE calculated with our DLA models are shown in Fig. 3 as functions of temperature 

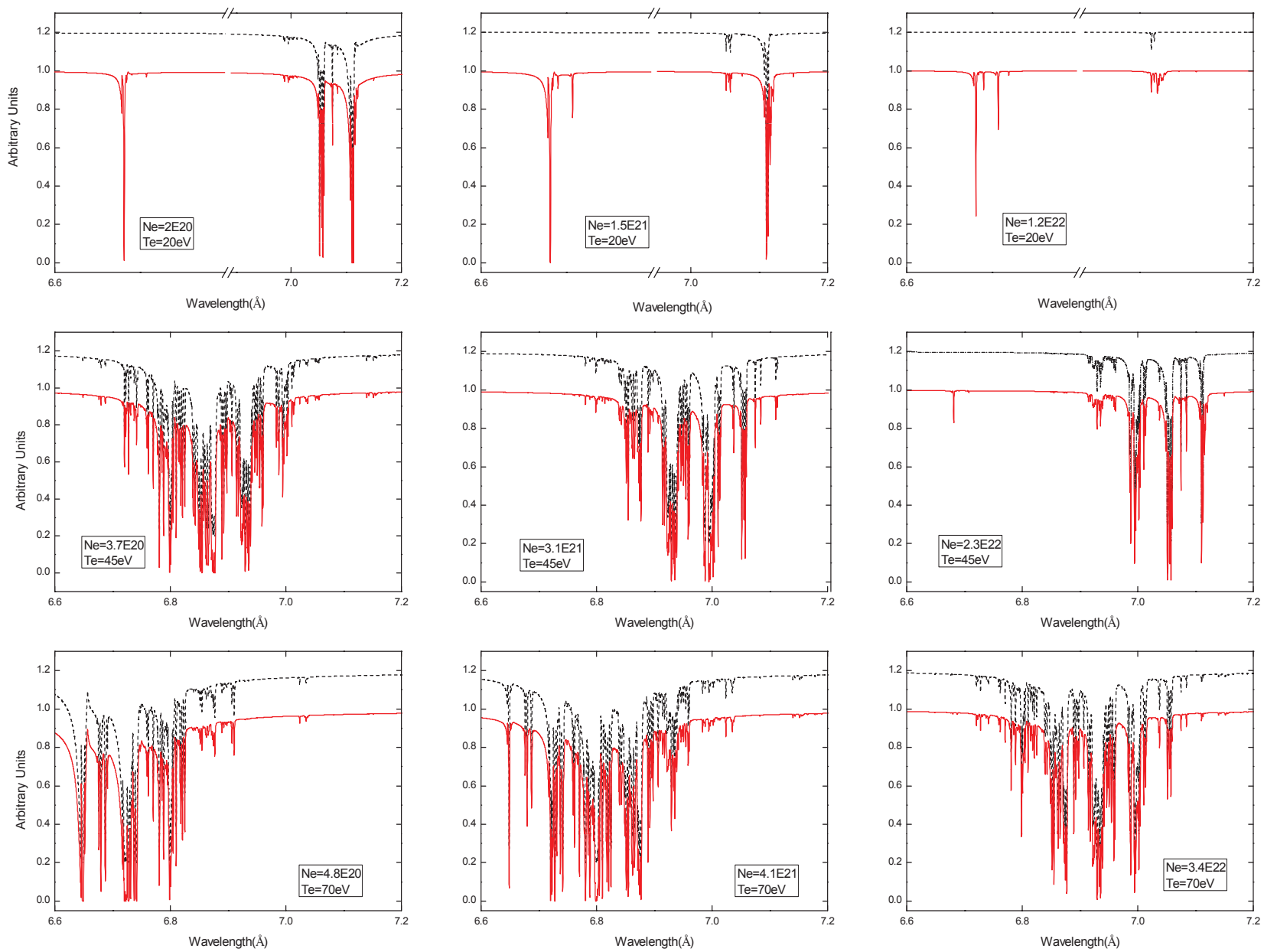

Fig. 3. K-shell absorption spectra calculated at different temperatures and densities. The electron temperature (in $\mathrm{eV}$ ) and density (in $\mathrm{cm}^{-3}$ ) are labeled in each panel. In all panels the spectra with M-shell ions included are the solid lines, while the spectra without M-shell ions included are dashed lines.

and electron density. We calculated the spectra at temperatures of $20 \mathrm{eV}, 45 \mathrm{eV}$, and $70 \mathrm{eV}$, and at densities of orders from $\sim 10^{20} \mathrm{~cm}^{-3}$ to $\sim 10^{22} \mathrm{~cm}^{-3}$, which can currently be achieved in the opacity experiments. The K-shell spectra of silicon that include M-shell ions are compared with the spectra that do not include M-shell ions in Fig. 3. For clarity, the spectra that do not account for M-shell ions have been shifted up in each panel. We neglected the instrumental broadenings at present, and kept the area density constant in the different calculations. As shown in the lower panels, it is found that, at high temperatures, the spectra do not vary whether the lowly ionized silicon ions are included or not, even if the density increases by two orders of magnitude. This result is expected since the ionization energy for $\mathrm{Si} \mathrm{I}(8.15 \mathrm{eV})$ to $\mathrm{Si}$ IV $(45.14 \mathrm{eV})$ are all lower than $70 \mathrm{eV}$, thus, the lowly ionized ions fully ionize and cannot exist. At moderate temperatures, as shown in the middle panels in Fig. 3, the temperature is still higher than the ionization energy for most of the lowly ionized $\mathrm{Si}$ ions and the contributions from these ions to the absorption spectra are also weak; however, at relatively high density, these contributions begin to be distinct as shown in the middle right panel in Fig. 3. In this panel, large differences in the wavelength around $6.68 \AA$ and $7.11 \AA$ can clearly be seen. The strong absorption features found around $6.68 \AA$ originate from the Ne-like $\mathrm{Si} \mathrm{V}$ ions, which also contributes to the absorption around 7.11 $\AA$. Under this condition, the effects of the lowly ionized silicon ions should be taken into account when modeling the K-shell spectra of Si. It is found that, at low temperatures, as shown in the upper panels in Fig. 3, the absorptions mainly originate from the lowly ionized ions. Especially at high densities, almost all the absorptions come from lowly ionized ions (upper right panel), namely from F-like to $\mathrm{Mg}$-like ions, and the absorptions from M-shell ions occupy more than $90 \%$.

In Fig. 4, the absorption spectra from each single ion were presented at a temperature of $20 \mathrm{eV}$ and density of $1.2 \times$ $10^{22} \mathrm{~cm}^{-3}$, respectively. The main absorption features originate from the Ne-like Si V and Mg-like Si III. Behar \& Netzer (2002) provided the K-shell atomic data for the strongest transitions only, however, we find that this is not enough when calculating the absorption spectra for the M-shell ions for some conditions. For example, the strongest absorption feature around $6.68 \AA$ is not the strongest transition among all Si V K-shell transitions. This is because the lower energy level for this transition is the ground state and has the largest population. Omission of these weak transitions in calculations causes fewer absorption features and the opacity. In summary, our results indicate that contributions from these lowly ionized ions of Si to the K-shell spectra are very important, and even can be dominant at low temperatures. However, it is a pity that we cannot compare our calculated results directly with those from OPAL because only Rosseland-mean opacities are provided by the OPAL databases. The monochromatic opacities from OP are only available in a limited photon energy range, and they are not included in the 


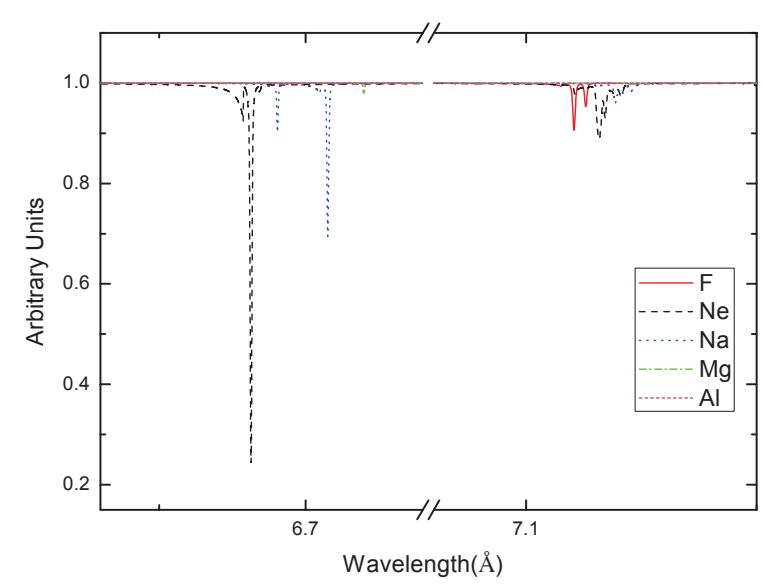

Fig. 4. K-shell absorption spectra for each ion of $\mathrm{Si}$ at a temperature of $20 \mathrm{eV}$ and density of $1.2 \times 10^{22} \mathrm{~cm}^{-3}$.

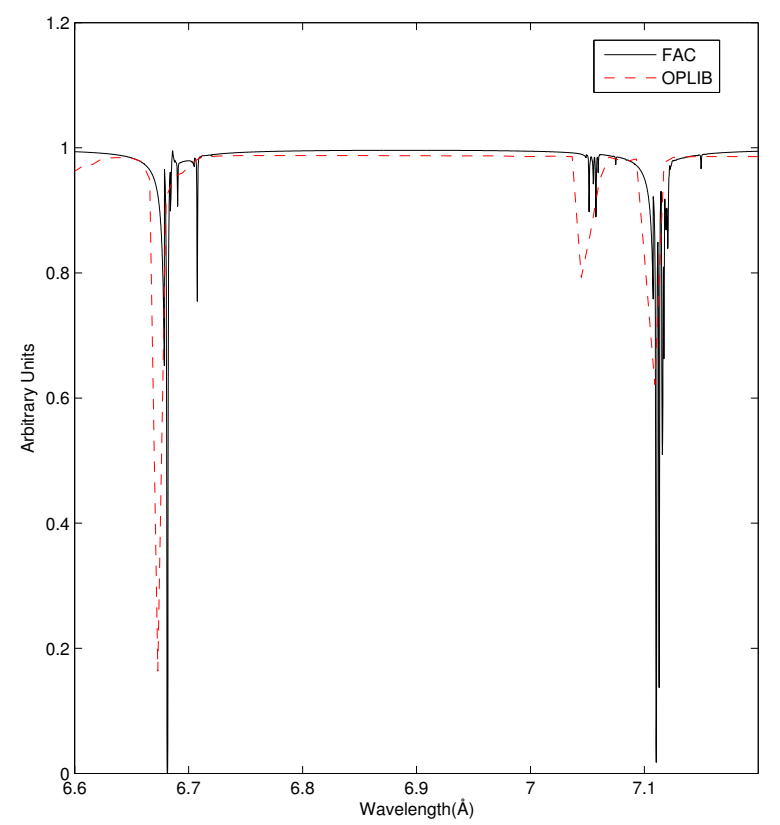

Fig. 5. K-shell absorption spectra of $\mathrm{Si}$ at a temperature of $20 \mathrm{eV}$ and density of $1.5 \times 10^{21} \mathrm{~cm}^{-3}$. The solid line is our FAC calculations and the dashed line is the OPLIB calculations.

present calculated spectra. Very recently, Colgan et al. (2016) published a new set of Los Alamos OPLIB opacity tables. In their work, transitions from the inner shell are included for all ion states. Through the inclusion of these inner-shell transitions, Colgan et al. (2016) find that improvements of solar models can be achieved with these new opacity tables. We compared our results with the OPLIB calculations in Fig. 5 at a temperature of $20 \mathrm{eV}$ and density of $1.5 \times 10^{21} \mathrm{~cm}^{-3}$. The two absorption spectra agree well in general while some differences are also distinct. Owing to the energy grid resolution, the detailed line structures are merged together in the spectra calculated from OPLIB monochromatic opacity. The central positions of absorption lines are also shifted in the two spectra, which is due to the differences between the adopted atomic data. The OPLIB absorptions at the wavelength around $7.045 \AA$ are stronger than that of our FAC calculations. Besides the differences in the adopted atomic data, Colgan et al. (2016) included more inner-shell transitions, for example, inner-shell transitions with valence electron $n$ up to 10 .

\subsection{Effects of different atomic data}

Using the HFR (Cowan 1981) and AUTOSTRUCTURE (Badnell 1986, 1997) codes, Palmeri et al. (2008) have computed the extensive atomic data for $\mathrm{Si}$ isonuclear sequences. These authors included relativistic corrections in their calculations, while we calculated the atomic data with FAC. Thus, it is interesting to investigate the effects of different atomic data on the Si K-shell absorption spectra. In our calculations of the absorption spectra, all the parameters are the same except for the atomic data, which means that the populations of ions and line broadening are the same for the same transitions in these two models.

Figure 6 showed the calculated spectra, and good agreements between the spectra calculated with our FAC data and those from Palmeri et al. (2008) can be found. Especially, the most absorption features are well reproduced both at high and low temperatures. However, some differences are also noticeable. For example, at low temperatures $(\sim 20 \mathrm{eV})$ two absorption lines from F-like Si VI are around 7.110 ̊ and 7.113 $\AA$ when adopting our FAC data, while using the data from Palmeri et al. (2008) these two lines are shifted to $7.122 \AA$ and $7.125 \AA$, respectively. The wavelength differences are about $12 \mathrm{~m} \AA$, which is just within the accuracy of our FAC data. The wavelength differences between our FAC results and the HFR results are still large with a range of $10 \mathrm{~m} \AA$ to $20 \mathrm{~m} \AA$ at moderate temperatures $(\sim 45 \mathrm{eV})$, and these wavelength differences get smaller at high temperatures $(\sim 70 \mathrm{eV})$. This is because although the main contributors to the spectra are still from lowly ionized ions at these relatively low temperatures, the uncertainties for the wavelengths or radiative rates of the $\mathrm{K}$-shell atomic data generally increase as the ion electron numbers increase (Palmeri et al. 2008). Another reason is that Palmeri et al. (2008) performed the wavelength corrections empirically for all ions except the M-shell ions in their $\mathrm{K}$-shell atomic data, which could also lead to a relative large difference for the data from M-shell ions. The differences in the line intensities are also obvious, as we show in an enlarged view of the absorption spectra in longer wavelength in Fig. 6. We noticed that the detailed absorption line intensities are different as the uncertainties of the oscillator strengths (linked to radiative rates) can be large. Indeed, the accuracy of the atomic data near the neutral ends of isonuclear sequences can be relatively low because of the increasing importance of the correlation effects causing strong mixings of the atomic configurations. Thus, it is comprehensible that different opacity database usually have large differences at low temperatures, which is the result of the lack of accurate atomic data, especially for the M-shell ions.

\section{Conclusions}

We developed a DLA model in LTE conditions to investigate the effects of the silicon M-shell ions on the absorption spectra. Our results show that at relatively low temperature $(\leq 45 \mathrm{eV})$ contributions from the lowly ionized ions to the absorption spectra are gradually increasing and may be dominant at lower temperatures, although their contributions can be neglected at high temperatures. We compared our results with the OPLIB databases and achieved good agreements. Comparisons of the calculated results by adopting different atomic data suggests that the detailed absorption features, such as line position and intensity, have large differences at low temperatures. This indicates that accurate measurements are needed to benchmark these theoretical atomic data.

Moreover, these effects are not unique to $\mathrm{Si}$, but appear to be a common phenomenon. As stated above, the opacity from 

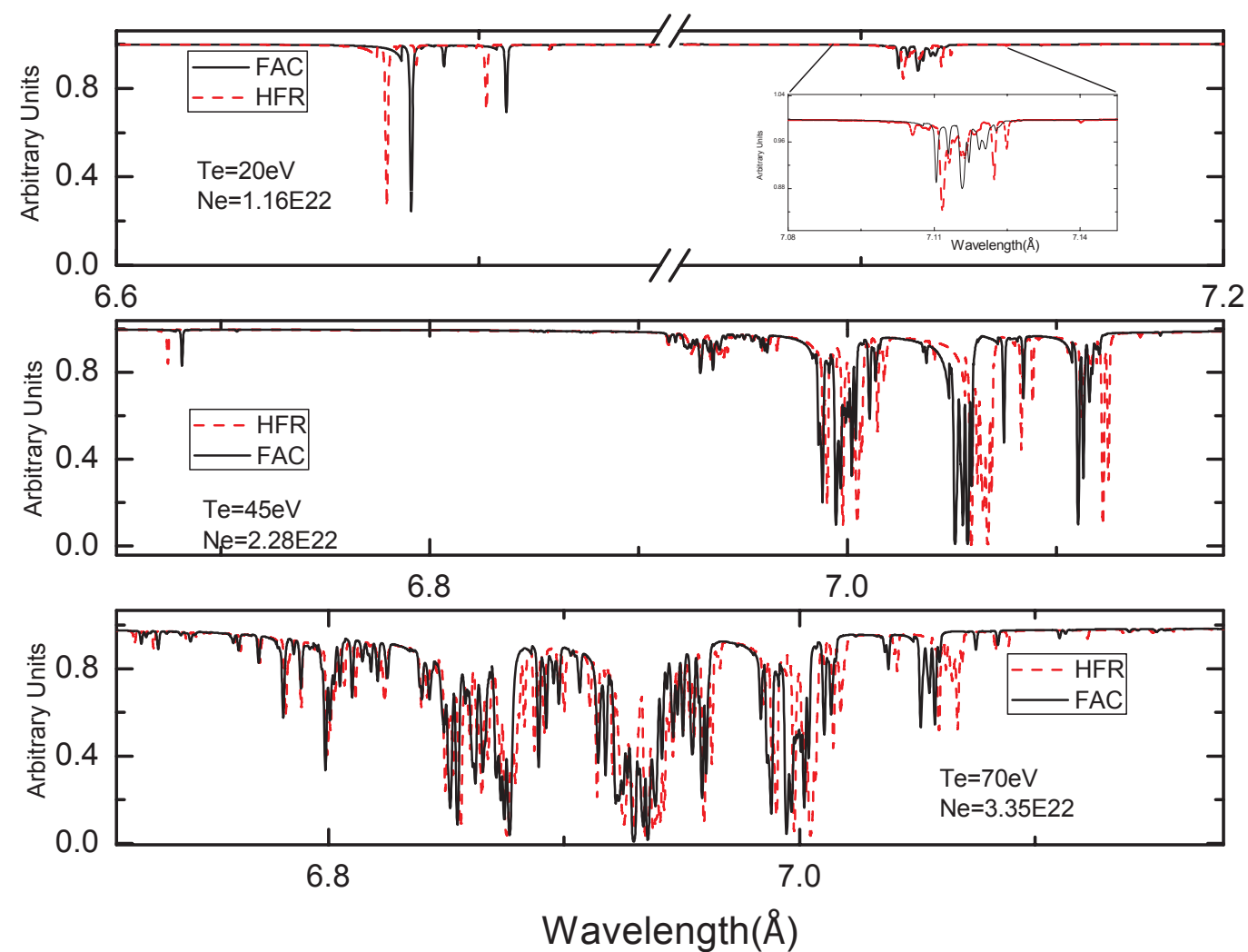

Fig. 6. K-shell absorption spectra calculated with our FAC atomic data and HFR from Palmeri et al. (2008). The electron temperature (in eV) and density $\left(\right.$ in $\mathrm{cm}^{-3}$ ) are indicated in each panel. The expanded spectra, indicated in the panel, are shown in the inset.

the database OP have already been claimed that some transitions have been neglected. Our result shows that the K-shell transitions from those M-shell ions could be potential candidates. Future investigations of these effects for Fe will be carried out soon.

Acknowledgements. This work is supported by National Basic Research Program of China (973 Program) under grant No. 2013CBA01503, and the National Natural Science Foundation of China under grants Nos. 11103040, 11473033, U1331122, $11173032,11573040$.

\section{References}

Badnell, N. R. 1986, J. Phys. B, 19, 3827

Badnell, N. R. 1997, J. Phys. B, 30, 1

Badnell, N. R., \& Seaton, M. J. 2004, J. Phys. B, 36, 4367

Badnell, N. R., Bautista, M. A., Butler, K., et al. 2005, MNRAS, 360, 458

Bailey, J. E., Rochau, G. A., Iglesias, C. A., et al. 2007, Phys. Rev. Lett., 99, 265002

Bailey, J. E., Rochau, G. A., Mancini, R. C., et al. 2009, Phys. Plasmas, 16, 058101

Bailey, J. E., Nagayama, T., Loisel, G., et al. 2015, Nature, 517, 56

Bautista, M. A., Mendoza, C., Kallman, T. R., \& Palmeri, P. 2003, A\&A, 403, 339

Bautista, M. A., Mendoza, C., Kallman, T. R., \& Palmeri, P. 2004, A\&A, 418, 1171

Behar, E., \& Netzer, H. 2002, ApJ, 570, 165

Beiersdorfer, P. 2009, Phys. Scr., T134, 014010

Beiersdorfer, P., Bitter, M., May, M. J., \& Roquemore, L. 2003, Rev. Sci. Instrum., 74, 1974

Chenais-Popovics, C., Merdji, H., Missalla, T., et al. 2000, ApJ, 127, 275

Colgan, J., Kilcrease, D. P., Magee, N. H., et al. 2016, ApJ, 817, 116

Cowan, R. D. 1981, The Theory of Atomic Structure and Spectra (Berkeley: Univ. California Press)

Da Silva, L. B., MacGowan, B. J., Kania, D. R., et al. 1992, Phys. Rev Lett., 69, 438

Davidson, S. J., Foster, J. M., Smith, C. C., et al. 1988, Appl. Phys. Lett., 52, 847

Gabriel, A. H., \& Jordan, C. 1969, MNRAS, 145, 241

Gu, M. F. 2003, ApJ, 582, 1241
Holczer, T., Behar, E., \& Kaspi, S. 2007, ApJ, 663, 799

Jin, F. T., Zeng, J. L., Huang, T. X., et al. 2009, ApJ, 693, 597

Kallman, T., Evans, Daniel, A., Marshall, H., et al. 2014, ApJ, 780, 121

Kaspi, S., Brandt, W. N., Netzer, H., et al. 2000, ApJ, 535, L17

Krongold, Y., Nicastro, F., Brickhouse, N. S., et al. 2003, ApJ, 597, 832

Massacrier, G., Artru, M. C. 2012, A\&A, 538, A52

Mendoza, C., Kallman, T. R., Bautista, M. A., Palmeri, P. 2004, A\&A, 414, 377

Nagayama, T., Bailey, J. E., Loisel, G., et al. 2012, Phys. Plasmas, 21, 056502

Palmeri, P., Mendoza, C., Kallman, T. R., \& Bautista, M. A. 2002, ApJ, 577, L119

Palmeri, P., Mendoza, C., Kallman, T. R., \& Bautista, M. A. 2003, A\&A, 403, 1175

Palmeri, P., Quinet, P., Mendoza, C., et al. 2008, ApJS, 177, 408

Palmeri, P., Quinet, P., Mendoza, C., et al. 2011, A\&A, 525, A59

Palmeri, P., Quinet, P., Mendoza, C., et al. 2012, A\&A, 543, A44

Perry, T. S., Davidson, S. J., Serduke, F. J. D., et al. 1991, Phys. Rev. Lett., 67, 3784

Porquet, D., \& Dubau, J. 2000, A\&AS, 143, 495

Porquet, D., Mewe, R., Dubau, J., et al. 2001, A\&A, 376, 1113

Porquet, D., Dubau, J., \& Grosso, N. 2010, Space Sci. Rev., 157, 103

Rochau, G. A., Bailey, J. E., \& MacFarlane, J. J. 2005, Phys. Rev. E, 72, 066405

Rogers, F. J., \& Iglesias, C. A. 1992, ApJS, 79, 507

Sako, M., Kahn, S. M., Behar, E., et al. 2001, A\&A, 365, L168

Sim, S. A., Miller, L., Long, K. S., Turner, T. J., \& Reeves, J. N. 2010, MNRAS, 404, 1369

Springer, P. T., Fields, D. J., Wilson, B. G., et al. 1992, Phys. Rev. Lett., 69, 3735

Turner, T. J., \& Miller, L. 2009, A\&ARv, 17, 47

Wei, H. G., Shi, J. R., Zhao, G., et al. 2008, ApJ, 683, A577

Wei, H. G., Shi, J. R., Zhao, G., et al. 2010, A\&A, 522, A103

Wei, H. G., Shi, J. R., Wang, F. L., et al. 2014, A\&A, 566, A105

Winhart, G., Eidmann, K., Iglesias, C. A., et al. 1995, J. Quant. Spectrosc. Radiat. Trans., 54, 437

Winhart, G., Eidmann, K., Iglesias, C. A., \& Bar-Shalom, A. 1996, Phys. Rev. E, 53, R1332

Witthoeft, M. C., Bautista, M. A., Mendoza, C., et al. 2009, ApJS, 182, 127

Xiong, G., Yang, J. M., Zhang, J. Y., et al. 2016, ApJ, 816, 36

Zeng, J. L., Zhao, G., \& Yuan, J. M. 2004, Phys. Rev. E, 70, 027401

Zhang, J., Li, H., Zhao, Y., et al. 2012, PhPl, 19, 113302 